\title{
Optically fabricated and controlled microtool as a mobile heat source in microfluidics
}

\author{
Engay, Einstom; Bunea, Ada-loana; Bañas, Andrew Rafael; Chouliara, Manto; Glückstad, Jesper
}

\section{Published in:}

Proceedings of SPIE

Link to article, DOI:

$10.1117 / 12.2325359$

Publication date:

2018

Document Version

Publisher's PDF, also known as Version of record

Link back to DTU Orbit

Citation (APA):

Engay, E., Bunea, A-I., Bañas, A. R., Chouliara, M., \& Glückstad, J. (2018). Optically fabricated and controlled microtool as a mobile heat source in microfluidics. In Proceedings of SPIE (Vol. 10804). [108040K] SPIE International Society for Optical Engineering. Proceedings of SPIE - The International Society for Optical Engineering https://doi.org/10.1117/12.2325359

\section{General rights}

Copyright and moral rights for the publications made accessible in the public portal are retained by the authors and/or other copyright owners and it is a condition of accessing publications that users recognise and abide by the legal requirements associated with these rights.

- Users may download and print one copy of any publication from the public portal for the purpose of private study or research.

- You may not further distribute the material or use it for any profit-making activity or commercial gain

- You may freely distribute the URL identifying the publication in the public portal 


\section{Optically fabricated and controlled microtool as a mobile heat source in microfluidics}

Einstom Engay, Ada-loana Bunea, Andrew Rafael Bañas, Manto Chouliara, Jesper Glückstad

Einstom Engay, Ada-loana Bunea, Andrew Rafael Bañas, Manto Chouliara, Jesper Glückstad, "Optically fabricated and controlled microtool as a mobile heat source in microfluidics," Proc. SPIE 10804, Advanced Manufacturing Technologies for Micro- and Nanosystems in Security and Defence, 108040K (8 October 2018); doi: 10.1117/12.2325359

SPIE. Event: SPIE Security + Defence, 2018, Berlin, Germany 


\title{
Optically fabricated and controlled microtool as a mobile heat source in microfluidics
}

\author{
Einstom Engay, Ada-Ioana Bunea, Andrew Rafael Bañas, Manto Chouliara and Jesper Glückstad* \\ DTU Fotonik, Department of Photonics Engineering, Technical University of Denmark, Ørsted \\ Plads 343, DK-2800 Kgs. Lyngby, Denmark \\ *jesper.gluckstad@fotonik.dtu.dk
}

\begin{abstract}
Microfluidic systems have gained much interest in the past decade as they tremendously reduce sample volume requirements for investigating different phenomena and for various medical, pharmaceutical and defense applications. Rapid heat transfer and efficient diffusive material transport are among the benefits of miniaturization. These have been achieved so far by tediously designing and fabricating application-specific microfluidic chambers or by employing microdevices that can be difficult to integrate in microfluidic systems. In this work, we present the fabrication and functionalization via two-photon polymerization and physical vapor deposition of microstructures that serve as heat sources in microfluidic devices upon laser illumination. In contrast to other existing methods that rely on photo-thermal effects, our microtools are amenable to optical manipulation and can be actuated in specific locations where heat generation is desired. Heating effects manifest in the presence of a temperature gradient, induced fluid flow and the formation of microbubbles.
\end{abstract}

Keywords: Two-photon polymerization, Thermoplasmonics, Optical trapping, Generalized Phase Contrast, Light Robotics

\section{INTRODUCTION}

Microfluidics has become an integral part of scientific investigations in the micro and nano- regimes and for medical examinations. Miniaturized microfluidic devices and lab-on-chip (LoC) systems allow cheaper and more efficient tests, as they only require minute amount of reagents and can significantly reduce analysis time. It remains a great challenge, however, to perform certain processes in microfluidic devices as opposed to macroscopic systems. For example, heating and mixing in a micro-environment requires additional microfabricated components and an external trigger attached to the microfluidic chamber. These components are often suitable only for specific predefined purposes and cannot be reconfigured once they are integrated in a certain microfluidic device. We are particularly interested in heating and micromixing as they provide understanding of fundamental phenomena at the microscale and are essential for several applications in PCR, material delivery and chemical agent determination.

Micro-mixing is difficult to achieve due to the inherently low Reynolds number in microfluids that results in laminar flows. To address this challenge, numerous methods have been reported. ${ }^{1,2}$ Passive mixers rely on the design of fluid channels that may include ridges and other parts to promote mixing as the fluid flows through the channel. Although passive mixers are easily fabricated, they often require additional pumps and are generally not reconfigurable. Additionally, once the passive mixer is fabricated, the user has minimal control over the fluid flow. On the other hand, active mixers rely on fabricated systems that are integrated into the microfluidic compartment. They are easily controlled by the user via an external driving source which can be an optical, acoustic or magnetic field. ${ }^{2}$ Natural convection promotes mixing in a slow process as it relies on density gradients. By harnessing temperature-programmed natural convection, micro-mixing was enhanced in a single microfluidic chamber by Kim et al. ${ }^{3}$ A heating instrument was fabricated and assembled in a microfluidic cartridge. By applying constant DC voltage alternately between two heating sites, a sufficient degree of mixing was achieved within a few tens of seconds.

Fabricated microtools have been employed to address challenges when dealing with microscopic systems. For example, microtools can act as mediator between a laser beam actuator and a biological specimen to minimize potential photodamage. ${ }^{4}$ The microtools can further be functionalized to achieve outcomes on-demand when externally triggered. Lighttriggered material delivery has been demonstrated using a hollow microtool embedded with a metal layer. ${ }^{5}$ Other elegant

Advanced Manufacturing Technologies for Micro- and Nanosystems in Security and Defence, edited by

Andrea Camposeo, Yuris Dzenis, Maria Farsari, Luana Persano, Proc. of SPIE Vol. 10804, 108040K

(C) 2018 SPIE · CCC code: $0277-786 X / 18 / \$ 18 \cdot$ doi: 10.1117/12.2325359 
applications include a micro-hand that simulates a grip when the structure is illuminated ${ }^{6}$ and a capsule-type microrobot with a pick-and-drop functionality for possible targeted drug delivery applications. ${ }^{7}$

In this work, we present the optical fabrication of microtools and their functionalization to enhance natural convection in straight microfluidic channels that, in turn, leads to enhanced local micro-mixing. The temperature increase needed for inducing convection is provided by increased light-heat conversion achieved by virtue of off-resonant plasmonic heating. The microtools were fabricated using a direct laser writing (DLW) method which offers a maskless, additive approach of 3D printing microstructures. Specifically, two-photon polymerization (2PP) was employed in this study using a femtosecond laser pulse tightly focused in a photosensitive resin to initiate polymerization within the beam's focal volume. ${ }^{8}$ The beam was then $3 \mathrm{D}$ scanned in the photoresist to trigger polymerization along the beam's path, allowing for the fabrication of microstructures. Once introduced in the microfluidic system, the microtools can be manipulated using optical trapping. $2 \mathrm{PP}$ has been previously employed for the fabrication of medical micro devices ${ }^{9}$ and microtools that can be used in different applications. ${ }^{5,10,11}$

\section{MATERIALS AND METHODS}

\subsection{Microtool fabrication and functionalization}

The microtools include three spherical trapping handles ( $8 \mu \mathrm{m}$ diameter) and a disk (10 $\mu \mathrm{m}$ diameter, $2 \mu \mathrm{m}$ thickness) as shown in Figure 1. The spherical handles facilitate optical trapping and manipulation and thus minimize possible drag effects, while the disk allows further functionalization to enhance light-heat conversion. The microtools were fabricated using a 2PP-based microfabrication system (Nanoscribe Photonics Professional GT, Nanoscribe GmbH, Germany), with Nanoscribe IPL-80 as photoresist on top of a glass substrate. To allow for selective coating, masks were printed on top of the body of the microtools leaving only the disk exposed. A set of 9 microtools with masks can be printed in 4 hours using the system. After fabrication, we immerse the substrate containing the printed microtools in isopropanol for 20 minutes to remove the uncrosslinked photoresist. Optical microscopy images of the microtools with and without masks are shown in Figure 2.

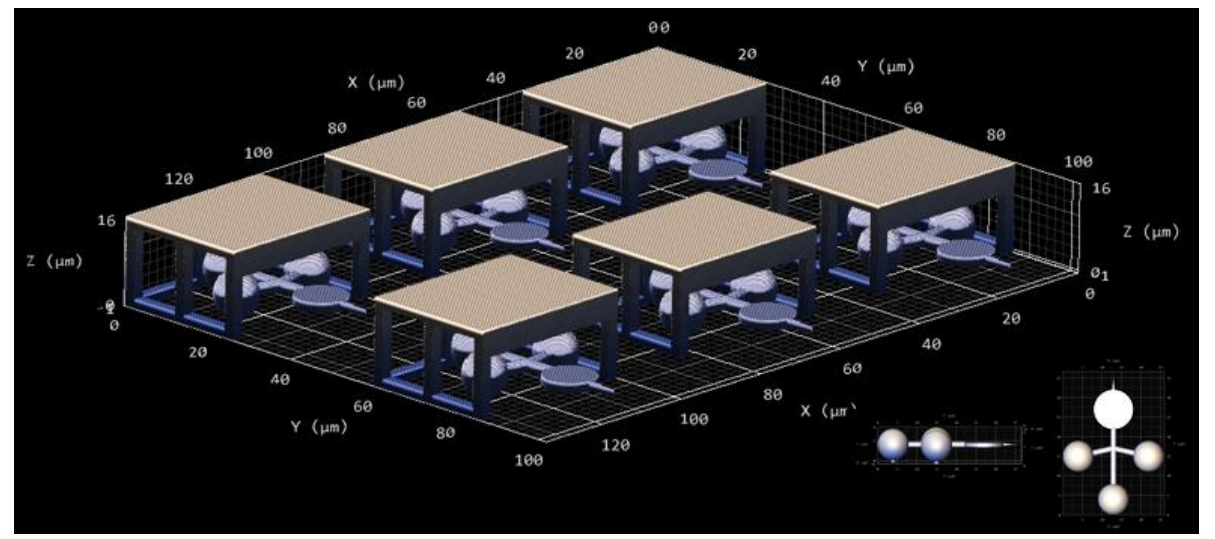

Figure 1. STL design of an array of microtools with masks. Inset (bottom right) shows the side and top view of the microtools without the mask.

After development, the microtools were functionalized by metal coating the disks via electron-beam physical vapor deposition (Wordentec QCL800). $1 \mathrm{~nm}$ layer of titanium was first deposited to act as an adhesive layer, followed by a 10 $\mathrm{nm}$ layer of gold. The deposited gold nanolayer was exploited for enhanced light absorption via plasmonic effects. The microtools were then detached from the glass substrate and transferred into a straight channel (Hellma cuvette with $250 \times 250 \mu \mathrm{m}^{2}$ inner cross section, $20 \mathrm{~mm}$ length) using a syringe with $100 \times 100 \mu \mathrm{m}^{2}$ square capillary tube. The solution inside the cuvette contained $0.125 \%$ polystyrene microspheres $\left(2 \mu \mathrm{m}\right.$ diameter Polybead ${ }^{\circledR}$ Microspheres, PolySciences, Inc.) and $1 \%$ Tween 80 in Milli-Q water $(18.2 \mathrm{M} \Omega \cdot \mathrm{cm})$. The microspheres serve as tracers to monitor the fluid flow inside the microfluidic channel. 

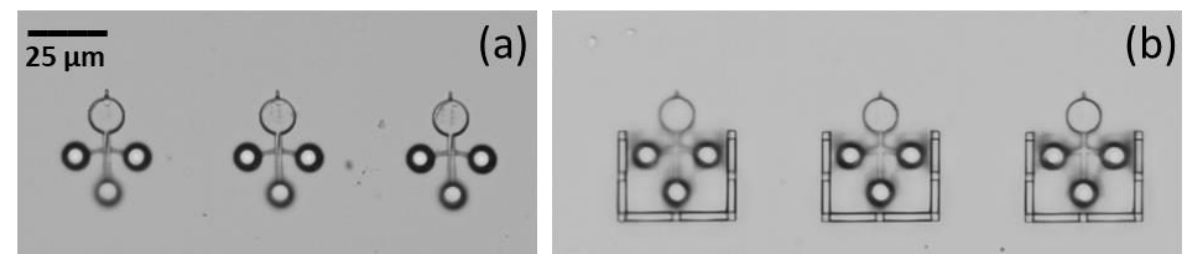

Figure 2. Fabricated microtool (a) without mask and (b) with mask for further functionalization via electron-beam physical vapor deposition.

\subsection{Optical manipulation and actuation}

Optical trapping and manipulation of the microtools was done using counterpropagating (CP) beams generated from a continuous wave $(\mathrm{CW})$ near-infrared (NIR) laser (IPG Photonics, $\lambda=1070 \mathrm{~nm}, 40 \mathrm{~W}$ maximum input power) through a proprietary illumination module based on Generalized Phase Contrast (GPC). The CP geometry does not require high numerical aperture (NA) objectives, in contrast to conventional optical tweezers. As such, a long working distance can be accommodated, allowing for side view imaging of the cuvette in addition to the traditional top view imaging. Figure 3 shows a schematic of the the optical trapping setup. A LabView-based graphical user interface (GUI) is used to control the relevant parameters for optical trapping and manipulation and to record images from the two cameras, $\mathrm{C} 1$ and $\mathrm{C} 2$. Live images from the top view camera are used to align the CP beams and to monitor the location of the microtools in the $\mathrm{x}-\mathrm{y}$ plane, transverse to the beam propagation. Optical traps are displayed as circular graphics overlaid on the top-view images. The microtool is moved in the transverse plane by simply dragging the trap overlays to the desired location. The side view camera is used to monitor the axial location of the microtools and to record the movement of the tracer microspheres for further data analysis. Axial manipulation is performed by changing the intensity ratio of the top and bottom beams in the GUI. For our experiments, three optical traps were generated to manipulate the microtools, while a fourth CP beam was used to illuminate the disk and thus activate the heating.

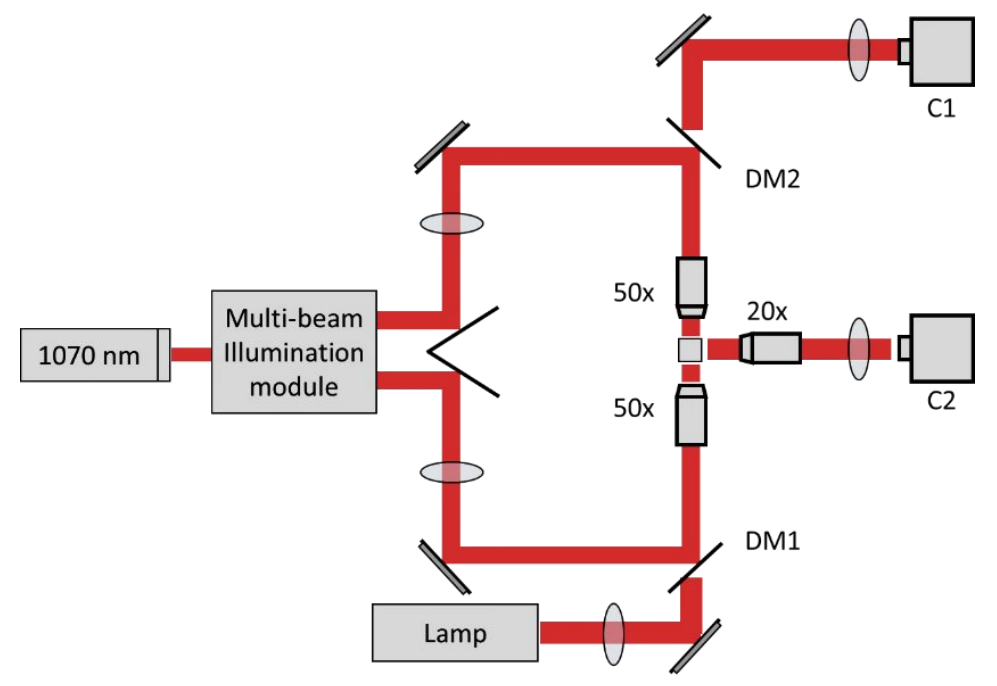

Figure 3. Optical trapping setup. A $1070 \mathrm{~nm}$ laser beam is incident on a proprietary illumination module that generates the counterpropagating optical traps. The top and bottom beams are then relayed to the sample using two opposing 50x objectives. The lower objective also collects broadband light for top view imaging through the top objective and C1 (CCD camera). A second lamp is installed (not shown in the figure) for the side view imaging through the 20x objective and $\mathrm{C} 2$.

\subsection{Data analysis}

Particle tracking was implemented for side-view videos recorded at 21 frames per second (fps) for a duration of $40 \mathrm{~s}$ ( 840 frames) starting from the time the heating beam was turned on. Tracking was performed using the Spot Tracking plugin in the ICY image analysis software. To minimize calculation errors due to out-of-plane displacements, only the particles that remain in-focus for at least $25 \mathrm{~s}$ (525 frames) were considered. The mean square displacements (MSDs) of the particles 
were then calculated from the first $25 \mathrm{~s}$ of all tracks using the freely available Matlab tool msdanalyzer. ${ }^{1}>$ From the MSD plots, the overall behavior of particle motion can be deduced. In the absence of significant fluid flow, the particles undergo random motion in which the MSD can be described by ${ }^{13,14}$

$$
\rho(t)=4 D t
$$

where $\rho(\mathrm{t})$ is the MSD at time $t$, and $D$ is the diffusion coefficient. On the other hand, for a directed and constrained flow such as that of natural convection, the MSD depends on the particle velocity $v$,

$$
\rho(t)=4 D t+v^{2} t^{2}
$$

To verify increased heat conversion with our coated microtools, a set of microtools with uncoated disks were also prepared and tested.

\section{RESULTS AND DISCUSSION}

Light to heat conversion as a result of illuminating the gold-coated disk with a laser beam manifested in three mechanisms: thermophoresis, natural convection and bubble formation. Upon illumination, the temperature in the immediate surrounding of the disk increased, inducing a thermal gradient in the system. Although we have not measured the temperature directly, we deduced the presence of thermal gradients from the motion of the particles present in the solution. For the case of the uncoated microtools, microspheres that were initially within the vicinity of the trapping handles and disks remained in those regions due to the optical gradient force introduced by the beams. Leaving the trapping and heating beams turned on for $240 \mathrm{~s}$ resulted in further aggregation of microspheres around the handles and disk. Meanwhile, illumination of the coated disks resulted in the immediate expulsion of the microspheres around the disk. This is a strong indication of the presence of a thermal gradient that causes the movement of particles from the hotter disk to the colder regions around it due to thermophoretic effects.
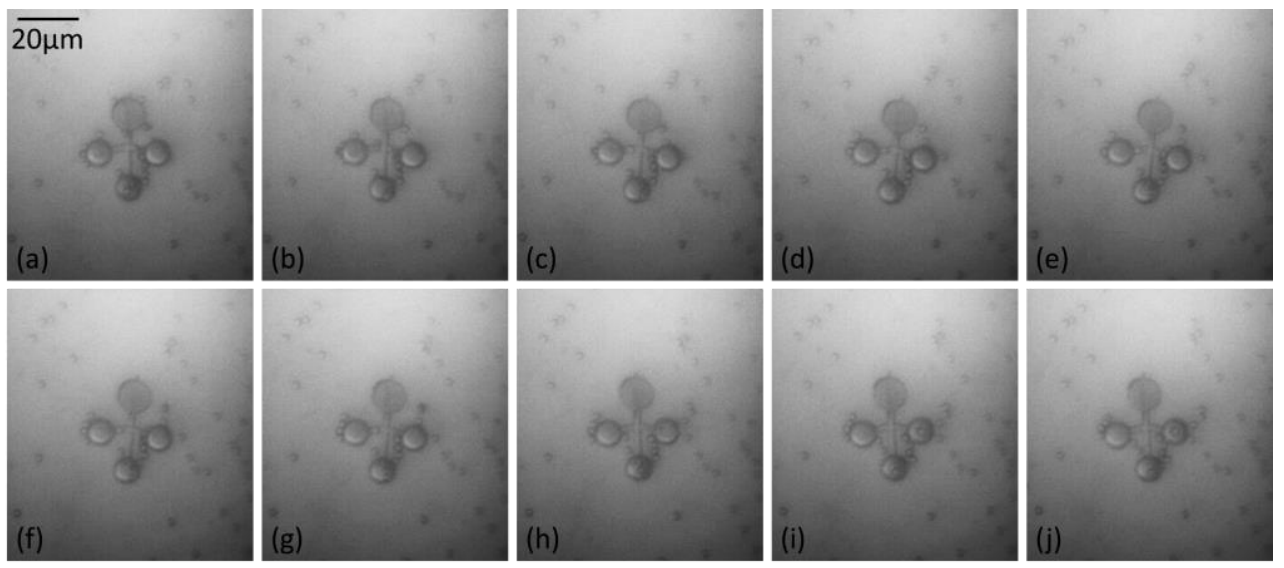

Figure 4. (a) Before the heating beam is turned on, (b)-(j) microspheres within the vicinity of the disk before illumination are expelled into the colder regions around the disk after the heating beam is turned on.

The heat generated by illuminating the coated disk was sufficient to effect global motion in the form of natural convection in the microfluidic chamber. From the side-view camera, a toroidal particle flow resembling that of Rayleigh-Bénard flow was directly observed. Microspheres that are initially beyond the side camera field of view move towards the microtool, and then to the top of the cuvette, and finally disperse away from the microtool. The average particle MSD plot generated for this case has a quadratic dependence with time. From the curve fit based on Eq. 2, the diffusion coefficient and mean particle velocity were calculated to be $D=2.74 \pm 0.11 \mu \mathrm{m}^{2} \mathrm{~s}^{-1}$ and $v=3.14 \pm 0.02 \mu \mathrm{m} \mathrm{s}^{-1}$, respectively. In contrast to this, the control experiments involving the uncoated tools produced a linear MSD plot. From Eq. $1, D=2.98 \pm 1.73 \mu \mathrm{m}^{2} \mathrm{~s}^{-1}$ 
for the uncoated microtool, which is in good agreement with the diffusion coefficient for the coated microtools. The linear behavior of $D$ indicates that the motion of the microspheres is dominated by Brownian motion, although sedimentation due to gravity would most likely have a substantial role for longer time periods. More importantly, illumination of the uncoated disk did not result in any significant global fluid flow, i.e. no natural convection.
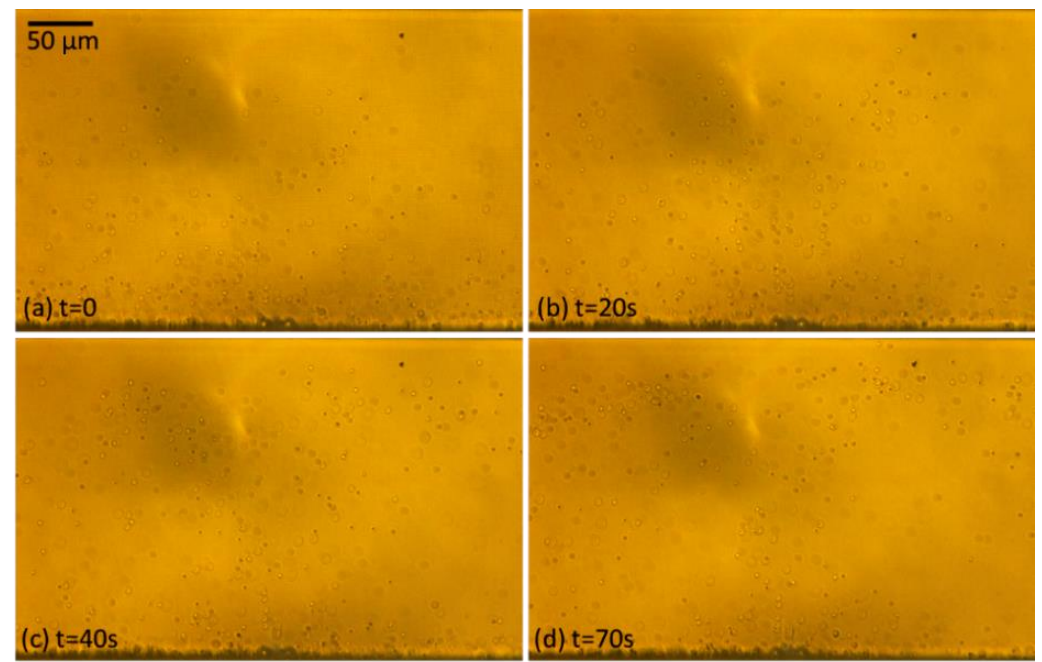

Figure 5. Natural convection induced by plasmon-mediated heating of gold-coated disk.

Finally, the generation of microbubbles was used to confirm plasmonic heating as a consequence of illuminating the goldcoated disk. The dynamics of bubble formation due to plasmonic heating has been extensively studied. ${ }^{1}>$ The relatively high power (tens of $\mathrm{mW}$ incident on the disk) employed in our experiments is required for rapid heating because we are relying on off-resonant thermoplasmonic heating. Resonant thermoplasmonic heating would require reduce laser power. Microbubbles promote micro-mixing, but in our case, the recoil on the microtool as the bubbles are generated overcomes the optical traps, therefore making the microtools very unstable.
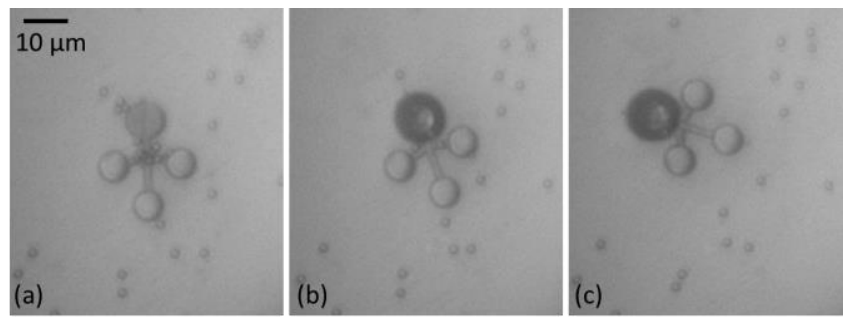

Figure 6. Bubble formation as a result of plasmonic heating. The microtool is destabilized from the optical traps when the bubble is formed.

\section{CONCLUSIONS}

We successfully fabricated microtools with disks using two-photon polymerization and further functionalized them by gold deposition. We integrated the microtools in a straight, closed microfluidic channel and manipulated them by optical trapping. With a laser beam that can be activated on-demand, the microtools facilitated the generation of heat manifesting in three mechanisms: thermophoresis, natural convection and bubble formation. Our fabricated microtools could potentially be used in applications that require localized heating and enhanced micro-mixing. 


\section{REFERENCES}

[1] Ward, K. and Fan, Z. H., "Mixing in microfluidic devices and enhancement methods," J. Micromechanics Microengineering 25(9) (2015).

[2] Campbell, C. J. and Grzybowski, B. A., "Microfluidic mixers: from microfabricate to selfassembling devices," Philiosophical Trans. R. Soc. A 362(1818), 1069-1086 (2004).

[3] Kim, S.-J., Wang, F., Burns, M. A. and Kurabayashi, K., "Temperature-Programmed Natural Convection for Micromixing and Biochemical Reaction in a Single Microfluidic Chamber," 4510-4516 (2009).

[4] Vizsnyiczai, G., Aekbote, B. L., Buzás, A., Grexa, I., Ormos, P. and Kelemen, L., "High accuracy indirect optical manipulation of live cells with functionalized microtools," Proc. IEEE 9922, 992216 (2016).

[5] Villangca, M. J., Palima, D., Bañas, A. R. and Glückstad, J., "Light-driven micro-tool equipped with a syringe function," Light Sci. Appl. 5(9), e16148 (2016).

[6] Martella, D., Nocentini, S., Nuzhdin, D., Parmeggiani, C. and Wiersma, D. S., "Photonic Microhand with Autonomous Action," Adv. Mater. 29(42), 1-8 (2017).

[7] Lee, S., Kim, S., Kim, S., Kim, J. Y., Moon, C., Nelson, B. J. and Choi, H., “A Capsule-Type Microrobot with Pick-and-Drop Motion for Targeted Drug and Cell Delivery," Adv. Healthc. Mater. 7(9), 1-6 (2018).

[8] Maruo, S., Nakamura, O. and Kawata, S., "Three-dimensional microfabrication with twophoton-absorbed photopolymerization," Opt. Lett. 22(2), 132-134 (1997).

[9] Son, A. I., Opfermann, J. D., McCue, C., Ziobro, J., Abrahams, J. H., Jones, K., Morton, P. D., Ishii, S., Oluigbo, C., Krieger, A., Liu, J. S., Hashimoto-Torii, K. and Torii, M., “An Implantable Micro-Caged Device for Direct Local Delivery of Agents," Sci. Rep. 7, 17624 (2017).

[10] Engay, E., Bunea, A.-I., Chouliara, M., Bañas, A. and Glückstad, J., "Natural convection induced by an optically fabricated and actuated microtool with a thermoplasmonic disk," Opt. Lett. 43(17) (2018).

[11] Glückstad, J. and Palima, D., [Light robotics : structure-mediated nanobiophotonics], Elsevier (2017).

[12] Tarantino, N., Tinevez, J. Y., Crowell, E. F., Boisson, B., Henriques, R., Mhlanga, M., Agou, F., Israël, A. and Laplantine, E., "Tnf and il-1 exhibit distinct ubiquitin requirements for inducing NEMO-IKK supramolecular structures,” J. Cell Biol. 204(2), 231-245 (2014).

[13] Michalet, X., "Mean square displacement analysis of single-particle trajectories with localization error: Brownian motion in an isotropic medium," Phys. Rev. E - Stat. Nonlinear, Soft Matter Phys. 82(4), 041914 (2010).

[14] Dunderdale, G., Ebbens, S., Fairclough, P. and Howse, J., "Importance of Particle Tracking and Calculating the Mean-Squared Displacement in Distinguishing Nanopropulsion from Other Processes," Langmuir 28(30), 10997-11006 (2012).

[15] Baffou, G., Polleux, J., Rigneault, H. and Monneret, S., "Super-heating and micro-bubble generation around plasmonic nanoparticles under cw illumination," J. Phys. Chem. C 118(9), 4890-4898 (2014). 